\title{
On the Spectral Characterization of $\boldsymbol{H}$-Shape Trees
}

\author{
Shengbiao Hu \\ Department of Mathematics, Qinghai Nationalities University, Xining, China \\ Email: shengbiaohu@aliyun.com
}

Received 6 March 2014; revised 7 April 2014; accepted 14 April 2014

Copyright (C) 2014 by author and Scientific Research Publishing Inc.

This work is licensed under the Creative Commons Attribution International License (CC BY). http://creativecommons.org/licenses/by/4.0/

c) (i) Open Access

\begin{abstract}
A graph $G$ is said to be determined by its spectrum if any graph having the same spectrum as $G$ is isomorphic to $G$. An $H$-shape is a tree with exactly two of its vertices having maximal degree 3 . In this paper, a formula of counting the number of closed 6-walks is given on a graph, and some necessary conditions of a graph $\Gamma$ cospectral to an $H$-shape are given.
\end{abstract}

\section{Keywords}

Spectra of Graphs, Cospectral Graphs, Spectra Radius, $H$-Shape Trees, Determined by Its Spectrum

\section{Introduction}

Let $G=(V, E)$ be a simple undirected graph with vertex set $V=\left\{v_{1}, v_{2}, \cdots, v_{n}\right\}$ and edge set $E$. Let $A(G)$ be the adjacency matrix of $G$. Since $A(G)$ is a real symmetric matrix, its eigenvalues must be real, and may be ordered as $\lambda_{1}(G) \geq \lambda_{2}(G) \geq \cdots \geq \lambda_{n}(G)$. The sequence of $n$ eigenvalues is called the spectrum of $G$, the largest eigenvalue $\lambda_{1}(G)$ is often called the spectral radius of $G$. The characteristic polynomial of $A(G)$ is called the characteristic polynomial of the graph $G$ and is denoted by $\varphi(G, \lambda)$.

Two graphs are cospectral if they share the same spectrum. A graph $G$ is said to be determined by its spetrum (DS for short) if for any graph $H, \varphi(H, \lambda)=\varphi(G, \lambda)$ implies that $H$ is isomorphic to $G$.

Determining what kinds of graphs are DS is an old problem, yet far from resolved, in the theory of graph spectra. Numerous examples of cospectral but non-isomorphic graphs are reported in literature [1]. However, there are few results known about DS graphs. For the background and some recent surveys of the known results about this problem and related topics, we refer the reader to [2]-[6] and references therein.

Because the kind of problems above are generally very hard to deal with, some more modest ones suggested by van Dam and Haemers [2], say, "Which trees are DS?”, this problem is also very hard to deal with, because we know a famous result of Schwenk [7], which says that almost all trees have non-isomorphic cospectral 
mates.

A $T$-shape $T\left(l_{1}, l_{2}, l_{3}\right)$ is a tree with exactly one of its vertices having maximal degree 3 such that $T\left(l_{1}, l_{2}, l_{3}\right)-v=p_{l_{1}} \cup p_{l_{2}} \cup p_{l_{3}}$, where $p_{l_{i}}$ is the path on $l_{i}(i=1,2,3)$ vertices, and $v$ is the vertex of degree 3 . More recently, Wang proved that $T$-shape tree $T\left(l_{1}, l_{2}, l_{3}\right)$ is DS; Wang and Xu [6] proved that $T$-shape tree $T\left(l_{1}, l_{2}, l_{3}\right)\left(l_{1} \leq l_{2} \leq l_{3}\right)$ is DS iff $\left(l_{1}, l_{2}, l_{3}\right) \neq(l, l, 2 l-2)$ for any positive integer $l \geq 2$.

An $H$-shape is a tree with exactly two of its vertices having maximal degree 3 . We denote by $H\left(l_{1}, l_{2}, l_{3}, l_{4}, l_{5}\right)\left(l_{1} \geq 0, l_{i} \geq 1, i=2,3,4,5\right)$ is an $H$-shape tree such that $H\left(l_{1}, l_{2}, l_{3}, l_{4}, l_{5}\right)-u-v=p_{l_{1}} \cup p_{l_{2}} \cup p_{l_{3}} \cup p_{l_{4}} \cup p_{l_{5}}$, and $H\left(l_{1}, l_{2}, l_{3}, l_{4}, l_{5}\right)-u=T\left(l_{1}, l_{4}, l_{5}\right)$, $H\left(l_{1}, l_{2}, l_{3}, l_{4}, l_{5}\right)-v=T\left(l_{1}, l_{2}, l_{3}\right) \cup p_{l_{4}} \cup p_{l_{5}}$, where $u$ and $v$ are the vertices of degree 3 .

In this paper, we give a formula of counting the number of closed 6-walks on a graph, and give some necessary conditions of a graph $\Gamma$ cospectral to an $H$-shape.

\section{Some Lemmas}

In the section, we will present some lemmas which are required in the proof of the main result.

Lemma 2.1 [8] The characteristic polynomial of a graph satisfies the following identities:

1) $\varphi\left(G_{1} \cup G_{2}, \lambda\right)=\varphi\left(G_{1}, \lambda\right) \varphi\left(G_{2}, \lambda\right)$,

2) $\varphi(G, \lambda)=\varphi(G-e, \lambda)-\varphi\left(G-v_{1} v_{2}, \lambda\right)$ if $e=v_{1} v_{2}$ is a cut-edge of $G$.

where $G-e$ denotes the graph obtained from $G$ by deleting the edge e and $G-v_{1} v_{2}$ denotes the graph obtained from $G$ by deleting the vertices $v_{1}, v_{2}$ and the edges incident to it.

Lemma 2.2 [1] Let $C_{n}, P_{n}$ denote the cycle and the path on $n$ vertices respectively. Then

$$
\begin{aligned}
& \varphi\left(C_{n}, \lambda\right)=\prod_{j=1}^{n}\left(\lambda-2 \cos \frac{2 \pi j}{n}\right)=2 \cos (n \arccos \lambda / 2)-2 \\
& \varphi\left(P_{n}, \lambda\right)=\prod_{j=1}^{n}\left(\lambda-2 \cos \frac{\pi j}{n+1}\right)=\frac{\sin ((n+1) \arccos \lambda / 2)}{\sin (\arccos \lambda / 2)}
\end{aligned}
$$

Let $\lambda=2 \cos \theta$, set $t^{1 / 2}=\mathrm{e}^{\mathrm{i} \theta}$, we get $\lambda=t^{1 / 2}+t^{-1 / 2}$, it is can be write the characteristic polynomial of $C_{n}$, $P_{n}$ in the following form [6]:

$$
\begin{gathered}
\varphi\left(C_{n}, t^{1 / 2}+t^{-1 / 2}\right)=t^{n / 2}+t^{-n / 2}-2=t^{-n / 2}\left(t^{n / 2}-1\right)^{2} \\
\varphi\left(P_{n}, t^{1 / 2}+t^{-1 / 2}\right)=t^{-n / 2}\left(t^{n+1}-1\right) /(t-1)
\end{gathered}
$$

Lemma 2.3 [4] [9] Let $\varphi(G, x)=\sum_{i=0}^{n} a_{i} \lambda^{n-i}$ be the characteristic polynomial of graph $G$ with $n$ vertices, then the coefficient of $\lambda^{n-i}$ is

$$
a_{i}=\sum_{\gamma}(-1)^{\operatorname{comp}(\gamma)} 2^{c y c(\gamma)}
$$

where $a_{0}=1$ and the sum is over all subgraphs $\gamma$ of $G$ consisting of disjoint edges and cycles, and having $i$ vertices. If $\gamma$ is such a subgraph then $\operatorname{comp}(\gamma)$ is the number of components in it and $\operatorname{cyc}(\gamma)$ is the number of cycles.

Lemma 2.4 [2] [10] Let $G$ be a graph. For the adjacency matrix, the following can be obtained from the spectrum.

1) The number of vertices.

2) The number of edges.

3) Whether $G$ is regular.

4) Whether $G$ is regular with any fixed girth.

5) The number of closed walk of any length.

6) Whether $G$ is bipartite. 


\section{Main Results}

The total number of closed k-walks in a graph G, denoted by $\left|w_{k}(G)\right|$.

Lemma 3.1 ([6] p. 657) Let $G$ be a graph with e edges, $x_{i}$ vertices of degree $i$, and $y$ 4-cycles. Then

$$
\left|w_{4}(G)\right|=2 e+4 \sum_{i}\left(\begin{array}{l}
i \\
2
\end{array}\right) x_{i}+8 y
$$

Lemma 3.2 Let $\Gamma$ be a graph with $n$ vertices. If $\Gamma$ cospectral to an $H$-shape and $\Gamma \neq W_{n}$, then

1) $\Gamma$ have the same degree sequences as the $H$-shape tree or $\Gamma$ have the degree sequences $(3,2,2, \cdots, 2,1,0)$.

2) $\Gamma$ contains no 4-cycles.

Proof. Let $\Gamma$ be a graph with $e$ edges, $x_{i}$ vertices of degree $i$, and $y$ 4-cycles. By lemma 2.4 we known that cospectral graphs have the same number of edges and closed 4-walks, respectively. Since $\Gamma$ is cospectral to an $H$-shape tree, hence by (4) we have

$$
2 e+4 \sum_{i}\left(\begin{array}{l}
i \\
2
\end{array}\right) x_{i}+8 y=6 n-2
$$

namely

$$
\sum_{i}\left(\begin{array}{l}
i \\
2
\end{array}\right) x_{i}+2 y=n=\sum_{i \geq 0} x_{i}
$$

Since

$$
\sum_{i \geq 2}(i-1) x_{i}=\sum_{i \geq 2} i x_{i}-\sum_{i \geq 2} x_{i}=\left(2 e-x_{1}\right)-\left(n-x_{0}-x_{1}\right)=2 e-n+x_{0}=n-2+x_{0},
$$

from (5), we have

$$
\sum_{i \geq 2}\left(\begin{array}{c}
i-1 \\
2
\end{array}\right) x_{i}+2 y=n-\sum_{i \geq 2}(i-1)=2-x_{0}
$$

the (7) imply to $y=1$ or 0 .

Case 1. $y=1$. by (7) we get $x_{0}=0$ and $x_{3}=x_{4}=\cdots=0$, by (5) we get $x_{2}=n-2$ and $x_{1}=2$, then $\Gamma=C_{4} \cup P_{n-2}$.

We known that "the spectrum of graph $W_{n}$ is the union of the spectra of the circuit $C_{4}$ and the path $P_{n-4}$ " [1], that is

$$
\varphi\left(W_{n}, \lambda\right)=\varphi\left(C_{4} \cup C_{4} \cup P_{n-4}, \lambda\right)
$$

Case 2. $y=0$. By (7) we have $x_{0} \leq 2$.

If $x_{0}=0$, then $x_{3}=2, x_{4}=x_{5}=\cdots=0$, by (5) we get $x_{2}=n-6$ and $x_{1}=4$. Thus $\Gamma$ have the same degree sequences as the $H$-shape tree.

If $x_{0}=1$, then $x_{3}=1, x_{4}=x_{5}=\cdots=0, x_{2}=n-3$ and $x_{1}=1$. The degree sequences of $\Gamma$ is $(3,2,2, \cdots, 2,1,0)$.

If $x_{0}=2$, then $x_{3}=x_{4}=\cdots=0, x_{2}=n,|V(\Gamma)| \geq n+2$, a contradiction.

Lemma 3.3 Let $G$ be a graph with $e$ edges, $x_{i}$ vertices of degree $i$, and $z 6$-cycles. Then

$$
\left|w_{6}(G)\right|=2 e+12 \sum_{i}\left(\begin{array}{l}
i \\
2
\end{array}\right) x_{i}+6 p_{4}+12 k_{1,3}+12 z
$$

where $p_{4}$ is the number of induced paths of length three and $k_{1,3}$ is the number of induced star $K_{1,3}$.

Proof. A close walk of length 6 can be produced from in the following five classes graphs, they are $P_{2}, P_{3}, P_{4}$, $K_{1,3}$ and $C_{6}$. For an edge and a 6-cycle, it is easy to see that the number of close 6-walks equals 2 and 12, respectively. For a $P_{3}$, the number of close 6-walks of a 1-degree vertex is 3 and the number of close 6 -walks of the 2-degree vertex is 6 , since the number of induced paths of length two is $\sum_{i}\left(\begin{array}{l}i \\ 2\end{array}\right) x_{i}$, hence for all induced paths $P_{3}$, the number of close 6-walks is $12 \sum_{i}\left(\begin{array}{l}i \\ 2\end{array}\right) x_{i}$. For a $P_{4}$, since the number of close 6-walks of a 1-degree ver- 
tex is 1 and the number of close 6-walks of a 2-degree vertex is 2, hence for all induced paths $P_{4}$, the number of close 6 -walks is $6 p_{4}$. Similarly, for a $K_{1,3}$, the number of close 6-walks of a 1-degree vertex is 2 and the number of close 6 -walks of the 3 -degree vertex is 6 , thus for all induced stars $K_{1,3}$, the number of close 6 -walks is $12 k_{1,3}$.

Corollary 3.4 Let $H=H\left(l_{1}, l_{2}, l_{3}, l_{4}, l_{5}\right)$, then

$$
\left|w_{6}(H)\right|=\left\{\begin{array}{l}
20 n+28-6 k\left(l_{1} \geq 1 \text { and have } k \text { elements are } 1 \text { in }\left\{l_{2}, l_{3}, l_{4}, l_{5}\right\}\right) \\
20 n+34-6 k\left(l_{1}=0 \text { and have } k \text { elements are } 1 \text { in }\left\{l_{2}, l_{3}, l_{4}, l_{5}\right\}\right)
\end{array}\right.
$$

where $0 \leq k \leq 4$.

Proof. Case 1. $l_{1} \geq 1$.

1) If $k=0$, that is $l_{i} \geq 2(i=2,3,4,5)$, then

$$
\left|w_{6}(H)\right|=2(n-1)+12[(n-6)+3 \times 2]+6\left[\left(l_{2}+l_{3}-2\right)+\left(l_{4}+l_{5}-2\right)+\left(l_{1}-1\right)+8\right]+12 \times 2=20 n+28
$$

where $\left(l_{2}+l_{3}-2\right),\left(l_{4}+l_{5}-2\right)$ and $\left(l_{1}-1\right)$ are the number of induced paths $P_{4}$ in $p_{l_{2}+l_{3}+1} \cdot p_{l_{4}+l_{5}+1}$ and $p_{l_{1}+2}$, respectively. The $8(=4+4)$ is the number of induced paths of through a 3-degree vertex $u($ or $v)$. If $P_{4}$ is such a induced path, then $u$ is an internal vertex in the $P_{4}$ and have at least a vertex in the $p_{l_{1}}$ (or $p_{l_{2}}$ ).

2) If $k \neq 0$, then

$$
\left|w_{6}(H)\right|=2(n-1)+12[(n-6)+3 \times 2]+6\left[\left(l_{2}+l_{3}-2\right)+\left(l_{4}+l_{5}-2\right)+\left(l_{1}-1\right)+(8-k)\right]+12 \times 2=20 n+28-6 k
$$

Case 2. $l_{1}=0$.

1) If $k \neq 0$, then

$$
\left|w_{6}(H)\right|=2(n-1)+12[(n-6)+3 \times 2]+6\left[\left(l_{2}+l_{3}-2\right)+\left(l_{4}+l_{5}-2\right)+8\right]+12 \times 2=20 n+34 .
$$

2) If $k=0$, similarly, we have $\left|w_{6}(H)\right|=20 n+34-6 k$.

Example 1. Let $H_{1}=H(0,1,1,1,1)$, by (9) we have

$$
\left|w_{6}\left(H_{1}\right)\right|=20 \times n+34-6 k=20 \times 6+34-6 \times 4=130,
$$

if we give to a suitable label for the $H_{1}$, by a simple calculation we can get the diagonal matrix of $A^{6}\left(H_{1}\right)$, that is

$$
\operatorname{diag}\left(A^{6}\left(H_{1}\right)\right)=[11,11,43,43,11,11]
$$

clearly, the sum of the elements in the diagonal matrix equals $4 \times 11+2 \times 43=130$.

Example 2. Let $H_{2}=H(2,2,2,2,2)$, by (9) we have $\left|w_{6}\left(H_{2}\right)\right|=20 \times 12+28=268$, similarly, if we give to a suitable label for the $H_{2}$, then we can get the diagonal matrix of $A^{6}\left(H_{2}\right)$, that is

$$
\operatorname{diag}\left(A^{6}\left(H_{2}\right)\right)=[6,6,6,6,22,22,22,22,29,29,49,49]
$$

clearly, the sum of the elements in the diagonal matrix equals $4 \times 6+4 \times 22+2 \times 29+2 \times 49=268$.

Lemma 3.5 Let $\Gamma$ be a graph with $n$ vertices, $e$ edges, $x_{i}$ vertices of degree $i$, and $z$ 6-cycles. If $\Gamma$ cospectral to $H\left(l_{1}, l_{2}, l_{3}, l_{4}, l_{5}\right)$ and $\Gamma \neq W_{\mathrm{n}}$, then

$$
2 \sum_{i \geq 2}\left(\begin{array}{c}
i-1 \\
2
\end{array}\right) x_{i}+p_{4}+2 k_{1,3}+2 z=\left\{\begin{array}{l}
n+9-k-2 x_{0}\left(l_{1} \geq 1 \text { and have } k \text { elements are } 1 \text { in }\left\{l_{2}, l_{3}, l_{4}, l_{5}\right\}\right) \\
n+10-k-2 x_{0}\left(l_{1}=0 \text { and have } k \text { elements are } 1 \text { in }\left\{l_{2}, l_{3}, l_{4}, l_{5}\right\}\right)
\end{array}\right.
$$

where $k(0 \leq k \leq 4)$ is the number of elements of equals 1 in $\left\{l_{2}, l_{3}, l_{4}, l_{5}\right\}$ and $p_{4}$ is the number of induced paths of length three and $k_{1,3}$ is the number of induced star $K_{1,3}$ in $\Gamma$.

Proof. If $l_{1} \geq 1$, by Lemma 3.3 we have

$$
\begin{aligned}
& 2 e+12 \sum_{i}\left(\begin{array}{l}
i \\
2
\end{array}\right) x_{i}+6 p_{4}+12 k_{1,3}+12 z=20 n+28-6 k, \quad 2 \sum_{i}\left(\begin{array}{l}
i \\
2
\end{array}\right) x_{i}+p_{4}+2 k_{1,3}+2 z=3 n+5-k, \\
& 2 \sum_{i \geq 2}\left(\begin{array}{c}
i-1 \\
2
\end{array}\right) x_{i}+p_{4}+2 k_{1,3}+2 z=3 n+5-2 \sum_{i \geq 2}(i-1) x_{i}=3 n+5-k-2\left(n-2+x_{0}\right)=n+9-k-2 x_{0} .
\end{aligned}
$$


Similarly, when $l_{1}=0$ the (10) hold.

Definition 1. Let $U$ be a graph obtained from a cycle $C_{\mathrm{g}}$ (g is even and $\left.6 \leq \mathrm{g} \leq n_{1}-2\right)$ and a path $P_{n_{1}-g}$, such that identifying an end vertex in the path and any one vertex in the cycle, and uniting an isolated vertex $K_{1}$.

If a graph have the degree sequences $(3,2,2, \cdots, 2,1,0)$, then the graph is $U$ uniting some cycle.

Lemma 3.6 Let $U^{\prime}$ be a graph with degree sequences $(3,2,2, \cdots, 2,1,0)$. If $U^{\prime}$ cospectral to an $H$-shape, then $U^{\prime}$ and $H$ satisfying one of the following conditions.

1) There are one 6-cycle in $U^{\prime}$ and $l_{1} \geq 1, l_{2}, l_{3}, l_{4}, l_{5} \geq 2$.

2) There are one 6-cycle in $U^{\prime}$ and $l_{1}=0$, have an element is 1 in $\left\{l_{2}, l_{3}, l_{4}, l_{5}\right\}$.

3) No 6-cycle in $U^{\prime}$ and $l_{1} \geq 1$, have two elements are 1 in $\left\{l_{2}, l_{3}, l_{4}, l_{5}\right\}$.

4) No 6-cycle in $U^{\prime}$ and $l_{1}=0$, have three elements are 1 in $\left\{l_{2}, l_{3}, l_{4}, l_{5}\right\}$.

Proof. Without loss of generality, Let $U^{\prime}=U \cup C_{n_{2}}$, where $n_{2}(\geq 6)$ is even and $n_{1}+n_{2}=n$. Let $U^{\prime}$ have $e$ edges, $x_{\mathrm{i}}$ vertices of degree $i$, and $z 6$-cycles.

Case 1. $l_{1} \geq 1$. By Lemma 3.5 we have $2 \times 1+\left[g+\left(n_{1}-g-3\right)+4+n_{2}\right]+2 \times 1+2 z=n+9-k-2,2 z=2-k$, get $k=0, z=1$ or $k=2, z=0$.

Case 2. $l_{1}=0$, we have $2 \times 1+\left[g+\left(n_{1}-g-3\right)+4+n_{2}\right]+2 \times 1+2 z=n+10-k-2,2 z=3-k$, get $k=1, z=$ 1 or $k=3, z=0$.

Lemma 3.7 Let $\lambda=t^{1 / 2}+t^{-1 / 2}$, then

$$
\begin{aligned}
\varphi & \left(H\left(l_{1}, l_{2}, l_{3}, l_{4}, l_{5}\right), t^{1 / 2}+t^{-1 / 2}\right) \\
= & \frac{t^{-n / 2}}{(t-1)^{5}}\left[(t-1)^{2}\left(t^{l_{1}+1}-1\right)\left(t^{l_{2}+l_{3}+2}-1\right)\left(t^{l_{4}+l_{5}+2}-1\right)-t(t-1)\left(t^{l_{1}}-1\right)\left(t^{l_{2}+l_{3}+2}-1\right)\left(t^{l_{4}+1}-1\right)\left(t^{l_{5}+1}-1\right)\right. \\
& \left.-t(t-1)\left(t^{l_{1}}-1\right)\left(t^{l_{2}+1}-1\right)\left(t^{l_{3}+1}-1\right)\left(t^{l_{4}+l_{5}+2}-1\right)+t^{2}\left(t^{l_{1}-1}-1\right)\left(t^{l_{2}+1}-1\right)\left(t^{l_{3}+1}-1\right)\left(t^{l_{4}+1}-1\right)\left(t^{l_{5}+1}-1\right)\right]
\end{aligned}
$$

Proof. By Lemma 2.1 (b) and Lemma 2.2 we have

$$
\begin{aligned}
\varphi & \left(H\left(l_{1}, l_{2}, l_{3}, l_{4}, l_{5}\right), \lambda\right) \\
= & \varphi\left(P_{l_{2}+l_{3}+1}, \lambda\right) \varphi\left(T\left(l_{1}, l_{4}, l_{5}\right), \lambda\right)-\varphi\left(P_{l_{2}}, \lambda\right) \varphi\left(P_{l_{3}}, \lambda\right) \varphi\left(T\left(l_{1}-1, l_{2}, l_{3}\right), \lambda\right) \\
= & \varphi\left(P_{l_{2}+l_{3}+1}, \lambda\right) \varphi\left(P_{l_{1}}, \lambda\right) \varphi\left(P_{l_{4}+l_{5}+1}, \lambda\right)-\varphi\left(P_{l_{2}+l_{3}+1}, \lambda\right) \varphi\left(P_{l_{1}-1}, \lambda\right) \varphi\left(P_{l_{4}}, \lambda\right) \varphi\left(P_{l_{5}}, \lambda\right) \\
& -\varphi\left(P_{l_{1}-1}, \lambda\right) \varphi\left(P_{l_{2}}, \lambda\right) \varphi\left(P_{l_{3}}, \lambda\right) \varphi\left(P_{l_{4}+l_{5}+1}, \lambda\right)+\varphi\left(P_{l_{1}-2}, \lambda\right) \varphi\left(P_{l_{2}}, \lambda\right) \varphi\left(P_{l_{3}}, \lambda\right) \varphi\left(P_{l_{4}}, \lambda\right) \varphi\left(P_{l_{5}}, \lambda\right) \\
& \varphi\left(H\left(l_{1}, l_{2}, l_{3}, l_{4}, l_{5}\right), t^{1 / 2}+t^{-1 / 2}\right) \\
= & \frac{t^{-n / 2}}{(t-1)^{5}}\left[(t-1)^{2}\left(t^{l_{1}+1}-1\right)\left(t^{l_{2}+l_{3}+2}-1\right)\left(t^{l_{4}+l_{5}+2}-1\right)-t(t-1)\left(t^{l_{1}}-1\right)\left(t^{l_{2}+l_{3}+2}-1\right)\left(t^{l_{4}+1}-1\right)\left(t^{l_{5}+1}-1\right)\right. \\
& \left.-t(t-1)\left(t^{l_{1}}-1\right)\left(t^{l_{2}+1}-1\right)\left(t^{l_{3}+1}-1\right)\left(t^{l_{4}+l_{5}+2}-1\right)+t^{2}\left(t^{l_{1}-1}-1\right)\left(t^{l_{2}+1}-1\right)\left(t^{l_{3}+1}-1\right)\left(t^{l_{4}+1}-1\right)\left(t^{l_{5}+1}-1\right)\right]
\end{aligned}
$$

If a graph has the same degree sequences as the $H$-shape, then $\Gamma$ is one of the following graphs $G_{1}, G_{2}, G_{3}, G_{4}$, $G_{5}$ in figure or it is an $H$-shape.

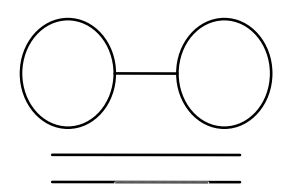

$\mathrm{G}_{1}$

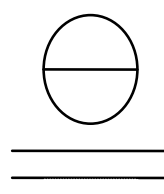

$\mathrm{G}_{2}$

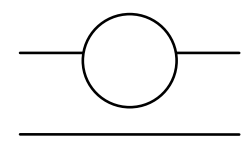

$\mathrm{G}_{3}$

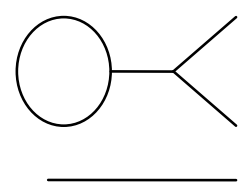

$\mathrm{G}_{4}$

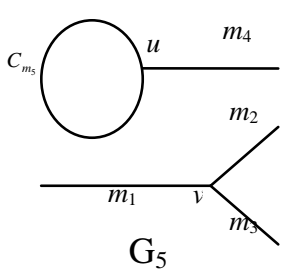

Lemma 3.8 If $\Gamma$ is cospectral to an $H$-shape tree, then $\Gamma$ contains no $P_{n_{1}} \cup P_{n_{2}}\left(n_{1}, n_{2}<n\right)$ as two connected component.

Proof. Assume that $\Gamma$ contains a $P_{n_{1}}$ as a connected component, by (11) some $l i$ is equal, without loss of generality, let $l_{1}=l_{2}=l_{4}=n_{1}$, then 


$$
\begin{aligned}
\varphi & \left(H\left(l_{1}, l_{2}, l_{3}, l_{4}, l_{5}\right), \lambda\right) \\
= & \varphi\left(P_{l_{1}+l_{3}+1}, \lambda\right) \varphi\left(P_{l_{1}+l_{5}+1}, \lambda\right)-\varphi\left(P_{l_{1}+l_{3}+1}, \lambda\right) \varphi\left(P_{l_{1}-1}, \lambda\right) \varphi\left(P_{l_{5}}, \lambda\right) \\
& -\varphi\left(P_{l_{1}-1}, \lambda\right) \varphi\left(P_{l_{3}}, \lambda\right) \varphi\left(P_{l_{1}+l_{5}+1}, \lambda\right)+\varphi\left(P_{l_{1}-2}, \lambda\right) \varphi\left(P_{l_{3}}, \lambda\right) \varphi\left(P_{l_{1}}, \lambda\right) \varphi\left(P_{l_{5}}, \lambda\right) \varphi\left(P_{l_{1}}, \lambda\right)
\end{aligned}
$$

If $\Gamma$ contains a $P_{n_{2}}$ as a connected component, then $l_{3}=l_{5}$ and $l_{1}+l_{3}+1=l_{1}$, a contradiction.

Thus, if a graph $\Gamma\left(\Gamma \neq W_{n}\right)$ cospectral to an $H$-shape and have the same degree sequences as the $H$-shape, then $\Gamma$ is one of the following graphs $G_{3}, G_{4}, G_{5}$ (Fig.) uniting some even cycle, respectively, or it is an $H$-shape.

Lemma 3.9 If $H_{1}=H\left(m_{1}, m_{2}, m_{3}, m_{4}, m_{5}\right)$ and $H=H\left(l_{1}, l_{2}, l_{3}, l_{4}, l_{5}\right)$ are cospectral, then $H\left(m_{1}, m_{2}, m_{3}, m_{4}, m_{5}\right) \cong H\left(l_{1}, l_{2}, l_{3}, l_{4}, l_{5}\right)$

Proof. By (11) we have

$$
\begin{aligned}
\varphi( & \left.H\left(l_{1}, l_{2}, l_{3}, l_{4}, l_{5}\right), t^{1 / 2}+t^{-1 / 2}\right) t^{n / 2}(t-1)^{5} \\
= & (t-1)^{2}\left(t^{l_{1}+1}-1\right)\left(t^{l_{2}+l_{3}+2}-1\right)\left(t^{l_{4}+l_{5}+2}-1\right)-t(t-1)\left(t^{l_{1}}-1\right)\left(t^{l_{2}+l_{3}+2}-1\right)\left(t^{l_{4}+1}-1\right) \\
& \left(t^{l_{5}+1}-1\right)-t(t-1)\left(t^{l_{1}}-1\right)\left(t^{l_{2}+1}-1\right)\left(t^{l_{3}+1}-1\right)\left(t^{l_{4}+l_{5}+2}-1\right)+t^{2}\left(t^{l_{1}-1}-1\right)\left(t^{l_{2}+1}-1\right)\left(t^{l_{3}+1}-1\right)\left(t^{l_{4}+1}-1\right)\left(t^{l_{5}+1}-1\right) \\
= & t^{\mathrm{n}+5}-4 t^{\mathrm{n}+4}+4 t^{\mathrm{n}+3}+t^{l_{1}+l_{2}+l_{3}+l_{4}+5}+t^{l_{1}+l_{2}+l_{3}+l_{5}+5}+t^{l_{1}+l_{2}+l_{4}+l_{5}+5}+t^{l_{1}+l_{3}+l_{4}+l_{5}+5}-2 t^{l_{1}+l_{2}+l_{3}+l_{4}+4} \\
& -2 t^{l_{1}+l_{2}+l_{3}+l_{5}+4}-2 t^{l_{1}+l_{2}+l_{4}+l_{5}+4}-2 t^{l_{1}+l_{3}+l_{4}+l_{5}+4}-t^{l_{2}+l_{3}+l_{4}+l_{5}+4}-t^{l_{1}+l_{2}+l_{3}+5}-t^{l_{1}+l_{4}+l_{5}+5}+2 t^{l_{1}+l_{2}+l_{3}+4} \\
& +2 t^{l_{1}+l_{4}+l_{5}+4}+t^{l_{2}+l_{3}+l_{4}+4}+t^{l_{2}+l_{3}+l_{5}+4}+t^{l_{2}+l_{4}+l_{5}+4}+t^{l_{3}+l_{4}+l_{5}+4}+t^{l_{1}+l_{2}+l_{4}+3}+t^{l_{1}+l_{2}+l_{5}+3}+t^{l_{1}+l_{3}+l_{4}+3} \\
& +t^{l_{1}+l_{3}+l_{5}+3}-t^{l_{2}+l_{4}+4}-t^{l_{2}+l_{5}+4}-t^{l_{3}+l_{4}+4}-t^{l_{3}+l_{5}+4}-2 t^{l_{2}+l_{3}+3}-2 t^{l_{4}+l_{5}+3}-t^{l_{1}+l_{2}+3}-t^{l_{1}+l_{3}+3}-t^{l_{1}+l_{4}+3} \\
& -t^{l_{1}+l_{5}+3}+t^{l_{2}+l_{3}+2}+t^{l_{4}+l_{5}+2}+t^{l_{1}+3}+2 t^{l_{2}+3}+2 t^{l_{3}+3}+2 t^{l_{4}+3}+2 t^{l_{5}+3}-t^{l_{2}+2}-t^{l_{3}+2}-t^{l_{4}+2}-t^{l_{5}+2}-4 t^{2}+4 t-1 \\
= & : \psi_{\mathrm{H}}(t)
\end{aligned}
$$

where $l_{1}+l_{2}+l_{3}+l_{4}+l_{5}+2=n$. By (14) we have

$$
\begin{aligned}
\varphi & \left(H\left(m_{1}, m_{2}, m_{3}, m_{4}, m_{5}\right), t^{1 / 2}+t^{-1 / 2}\right) t^{n / 2}(t-1)^{5} \\
= & t^{n+5}-4 t^{n+4}+4 t^{n+3}+t^{m_{1}+m_{2}+m_{3}+m_{4}+5}+t^{m_{1}+m_{2}+m_{3}+m_{5}+5}+t^{m_{1}+m_{2}+m_{4}+m_{5}+5}+t^{m_{1}+m_{3}+m_{4}+m_{5}+5} \\
& -2 t^{m_{1}+m_{2}+m_{3}+m_{4}+4}-2 t^{m_{1}+m_{2}+m_{3}+m_{5}+4}-2 t^{m_{1}+m_{2}+m_{4}+m_{5}+4}-2 t^{m_{1}+m_{3}+m_{4}+m_{5}+4}-t^{m_{2}+m_{3}+m_{4}+m_{5}+4} \\
& -t^{m_{1}+m_{2}+m_{3}+5}-t^{m_{1}+m_{4}+m_{5}+5}+2 t^{m_{1}+m_{2}+m_{3}+4}+2 t^{m_{1}+m_{4}+m_{5}+4}+t^{m_{2}+m_{3}+m_{4}+4}+t^{m_{2}+m_{3}+m_{5}+4} \\
& +t^{m_{2}+m_{4}+m_{5}+4}+t^{m_{3}+m_{4}+m_{5}+4}+t^{m_{1}+m_{2}+m_{4}+3}+t^{m_{1}+m_{2}+m_{5}+3}+t^{m_{1}+m_{3}+m_{4}+3}+t^{m_{1}+m_{3}+m_{5}+3}-t^{m_{2}+m_{4}+4} \\
& -t^{m_{2}+m_{5}+4}-t^{m_{3}+m_{4}+4}-t^{m_{3}+m_{5}+4}-2 t^{m_{2}+m_{3}+3}-2 t^{m_{4}+m_{5}+3}-t^{m_{1}+m_{2}+3}-t^{m_{1}+m_{3}+3}-t^{m_{1}+m_{4}+3} \\
& -t^{m_{1}+m_{5}+3}+t^{m_{2}+m_{3}+2}+t^{m_{4}+m_{5}+2}+t^{m_{1}+3}+2 t^{m_{2}+3}+2 t^{m_{3}+3}+2 t^{m_{4}+3}+2 t^{m_{5}+3}-t^{m_{2}+2}-t^{m_{3}+2} \\
& -t^{m_{4}+2}-t^{m_{5}+2}-4 t^{2}+4 t-1 \\
= & \psi_{H 1}(t)
\end{aligned}
$$

Let $H_{1}(t)=H(t)$, without loss of generality, we assume that $l_{2} \geq l_{3} \geq l_{4} \geq l_{5}$ and $m_{2} \geq m_{3} \geq m_{4} \geq m_{5}$. Comparing the $4^{\text {th }}$ lowest term of $H(t)$ and $H_{1}(t)$, we get $m_{5}=l_{5}$. Similarly, we comparing the $5^{\text {th }}, 6^{\text {th }}$ and $7^{\text {th }}$ lowest term of $H(t)$ and $H_{1}(t)$, respectively, we get $m_{4}=l_{4}, m_{3}=l_{3}$ and $m_{2}=l_{2}$. By $m_{1}+m_{2}+m_{3}+m_{4}+m_{5}+2=l_{1}+l_{2}+l_{3}+l_{4}+l_{5}+2=n$, we get $m_{1}=l_{1}$, thus $H\left(m_{1}, m_{2}, m_{3}, m_{4}, m_{5}\right) \cong H\left(l_{1}, l_{2}, l_{3}, l_{4}, l_{5}\right)$.

Lemma 3.10 Let $G_{5}$ be a graph in Figure, then $G_{5}$ and $H$-shape are not cospectral.

Proof. Let $G_{5}-u-v=P_{m_{1}} \cup P_{m_{2}} \cup P_{m_{3}} \cup P_{m_{4}} \cup P_{m_{5}-1}=1 \quad\left(m_{i} \geq 1, i=1,2,3,4 . m_{5} \geq 4\right)$, that is $m_{1}+m_{2}+m_{3}+m_{4}+m_{5}+1=n$. Denote the first component by $G_{5,1}$ and the second component by $G_{5,2}$. By Lemma 2.1 and Lemma 2.3 we have 


$$
\begin{gathered}
\varphi\left(G_{5,1}, \lambda\right)=\varphi\left(C_{m_{5}}, \lambda\right) \varphi\left(P_{m_{4}}, \lambda\right)-\varphi\left(P_{m_{5}-1}, \lambda\right) \varphi\left(P_{m_{4}-1}, \lambda\right) \\
\varphi\left(G_{5,1}, t^{1 / 2}+t^{-1 / 2}\right) \\
=t^{-m_{5} / 2}\left(t^{m_{5} / 2}-1\right)^{2} \frac{t^{-m_{4} / 2}}{t-1}\left(t^{m_{4}+1}-1\right)-\frac{t^{-\left(m_{4}-1\right) / 2}}{t-1}\left(t^{m_{4}}-1\right) \frac{t^{-\left(m_{5}-1\right) / 2}}{t-1}\left(t^{m_{5}}-1\right) \\
=\frac{t^{-\left(m_{4}+m_{5}\right) / 2}}{(t-1)^{2}}\left[(t-1)\left(t^{m_{5} / 2}-1\right)^{2}\left(t^{m_{4}+1}-1\right)-t\left(t^{m_{4}}-1\right)\left(t^{m_{5}}-1\right)\right], \\
\varphi\left(G_{5,2}, \lambda\right)=\varphi\left(P_{m_{1}}, \lambda\right) \varphi\left(P_{m_{2}+m_{3}+1}, \lambda\right)-\varphi\left(P_{m_{1}-1}, \lambda\right)\left(P_{m_{2}}, \lambda\right)\left(P_{m_{3}}, \lambda\right) \\
=\frac{t^{-m_{1} / 2}}{t-1}\left(t^{m_{1}+1}-1\right) \frac{\left.t^{-1 / 2}\right)}{t-1}\left(t^{m_{2}+m_{3}+2}-1\right)-\frac{t^{-\left(m_{1}-1\right) / 2}}{t-1}\left(t^{m_{1}}-1\right) \frac{t^{-m_{2} / 2}}{t-1}\left(t^{m_{2}+1}-1\right) \frac{t^{-m_{3} / 2}}{t-1}\left(t^{m_{3}+1}-1\right) \\
=\frac{t^{-\left(m_{1}+m_{2}+m_{3}+1\right) / 2}}{(t-1)^{2}}\left[\left(t^{m_{1}+1}-1\right)\left(t^{m_{2}+m_{3}+2}-1\right)-t\left(t^{m_{1}}-1\right)\left(t^{m_{2}+1}-1\right)\left(t^{m_{3}+1}-1\right)\right] .
\end{gathered}
$$

By Lemma 2.1 (a) we have

$$
\begin{aligned}
& \varphi\left(G_{5}, t^{1 / 2}+t^{-1 / 2}\right) t^{n / 2}(t-1)^{5} \\
& =\left[(t-1)\left(t^{m_{5} / 2}-1\right)^{2}\left(t^{m_{4}+1}-1\right)-t\left(t^{m_{4}}-1\right)\left(t^{m_{5}}-1\right)\right] \times\left[\left(t^{m_{1}+1}-1\right)\left(t^{m_{2}+m_{3}+2}-1\right)-t\left(t^{m_{1}}-1\right)\left(t^{m_{2}+1}-1\right)\left(t^{m_{3}+1}-1\right)\right] \\
& =: \psi_{G 5}(t)
\end{aligned}
$$

Comparing (14) and (15), since $\psi_{H}(0)=-1$ for any $l_{i}(i=1,2, \cdots, 5)$ and $\psi_{G 5}(0)=1$ for any $m_{i}(i=1,2, \cdots, 5)$, hence $\psi_{H}(t) \neq \psi_{G 5}(t) . G_{5}$ and $H$-shape are not cospectral.

Remark. If $G_{5}$ uniting some $C_{n_{i}}$, without loss of generality, let $G_{5,1}=G_{5} \cup C_{n-n_{1}}$, where $m_{1}+m_{2}+m_{3}+m_{4}$

$+m_{5}+1=n_{1}$. Since $\varphi\left(C_{n-n_{1}} t^{1 / 2}+t^{-1 / 2}\right)=t^{-\left(n-n_{1}\right) / 2}\left(t^{\left(n-n_{1}\right) / 2}-1\right)^{2}$, we have $\psi_{G 5,1}(t)=\psi_{G 5}(t)\left(t^{\left(n-n_{1}\right) / 2}-1\right)^{2}$, $\psi_{G 5,1}(0)=\psi_{G 5}(0)=1, \quad \psi_{H}(t) \neq \psi_{G 5,1}(t)$. Thus, $G_{5,1}$ and $H$-shape are not cospectral.

Theorem 3.11 Let $H=H\left(l_{1}, l_{2}, l_{3}, l_{4}, l_{5}\right) \quad\left(l_{1} \geq 0, l_{i} \geq 1, i=2,3,4,5\right)$, if a graph $\Gamma\left(\Gamma \neq W_{\mathrm{n}}\right)$ cospectral to an $H$-shape, then either $\Gamma$ is $U$ (Definition 1$)$ uniting some even cycles $C_{n_{i}}\left(n_{\mathrm{i}} \geq 6\right)$, denoted by $U^{\prime}$, and $U^{\prime}, H$ satisfying one of the following conditions.

1) There are one 6-cycle in $U^{\prime}$ and $l_{1} \geq 1, l_{2}, l_{3}, l_{4}, l_{5} \geq 2$.

2) There are one 6-cycle in $U^{\prime}$ and $l_{1}=0$, have 1 element is 1 in $\left\{l_{2}, l_{3}, l_{4}, l_{5}\right\}$.

3) No 6-cycle in $U^{\prime}$ and $l_{1} \geq 1$, have 2 elements are 1 in $\left\{l_{2}, l_{3}, l_{4}, l_{5}\right\}$.

4) No 6-cycle in $U^{\prime}$ and $l_{1}=0$, have 3 elements are 1 in $\left\{l_{2}, l_{3}, l_{4}, l_{5}\right\}$, or $\Gamma$ is the graph $G_{3}$ and $G_{4}$ in Figure uniting some even cycles $C_{n_{i}}\left(n_{i} \geq 6\right)$, respectively.

Proof. This result is contained from Lemma 3.2 up to Lemma 3.10.

\section{Funding}

This work is supported by the Natural Science Foundation of Qinghai Province (Grant No. 2011-Z-911).

\section{References}

[1] Cvetkovi'c, D., Doob, M. and Sachs, H. (1980) Spectra of Graphs-Theory and Application. Academic Press, New York.

[2] van Dam, E.R. and Haemers, W.H. (2003) Which Graph Are Determined by Their Spectrum? Linear Algebra and Its Applications, 373, 241-272. http://dx.doi.org/10.1016/S0024-3795(03)00483-X 
[3] Doob, M. and Haemers, W.H. (2002) The Complement of the Path Is Determined by Its Spectrum. Linear Algebra and Its Applications, 356, 57-65. http://dx.doi.org/10.1016/S0024-3795(02)00323-3

[4] Noy, M. (2003) Graphs Determined by Polynomial Invariants. Theoretical Computer Science, 307, 365-384.

[5] Smith, J.H. (1970) Some Propertice of the Spectrum of Graph. In: Guy, R., et al., Eds., Combinatorial Structure and Their Applications, Gordon and Breach, New York, 403-406.

[6] Wang, W. and Xu, C.-X. (2006) On the Spactral Characterization of T-Shape Trees. Linear Algebra and Its Applications, 414, 492-501. http://dx.doi.org/10.1016/j.laa.2005.10.031

[7] Schwenk, A.J. (1973) Almost All Trees Are Cospectral. In: Harary, F., Ed., New Directions in the Theory of Graphs, Academic Press, New York, 275-307.

[8] Godsil, C.D. (1993) Algebraic Combinatorics. Chapman \& Hall, New York.

[9] Sachs, H. (1964) Beziehungen zwischen den in einem graphen enthaltenen kreisenund seinem charakteristischen polynom. Publicationes Mathematicae, 11, 119-134.

[10] Omidi, G.R. and Tajbakhsh, K. (2007) Starlike Trees Are Determined by Their Laplacian Spectrum. Linear Algebra and Its Applications, 422, 654-658. http://dx.doi.org/10.1016/j.laa.2006.11.028 